\title{
MEMBRANE-DIALYZER INJECTION LOOP FOR ENHANCING THE SELECTIVITY OF ANION-RESPONSIVE LIQUID-MEMBRANE ELECTRODES IN FLOW SYSTEMS
}

Part 2. A Selective Sensing System for Salicylate

\author{
QINLIN CHANG and M. E. MEYERHOFF* \\ Department of Chemistry, University of Michigan, Ann Arbor, Michigan 48109 (U.S.A.)
}

(Received 23rd January 1986)

\section{SUMMARY}

An electrode-based flow-injection system suitable for the direct determination of salicylic acid is described. The system utilizes a tubular polymer membrane electrode based on manganese(III) tetraphenylporphyrin chloride to sense salicylate ions formed in a recipient buffer solution held within the upper channel of a flow-through membrane dialyzer assembly. Samples containing salicylic acid are manually introduced into the lower channel of the dialysis unit, in which a thin silicone rubber membrane separates the two channels. The analyte is trapped across the membrane as salicylate ions within a static layer of an appropriate recipient buffer. After a fixed trapping time, the recipient plug is flushed to the electrode in a conventional flow-injection manner. Peak potentials observed are logarithmically related to the salicylic acid concentrations in the original sample. Without the dialysis unit, the electrode response to salicylate is nearly Nernstian over the range $2 \times 10^{-6}-10^{-2} \mathrm{M}$. In the complete flow/dialysis system, near Nernstian response was achieved for $10^{-4}-10^{-2} \mathrm{M}$ salicylate with a 2-min trapping time. Detection limits can be altered by changing the trapping time. Anionic salicylate can be determined by acidifying the sample. The resulting system offers very high selectivity for salicylate (as salicylic acid) over most inorganic and organic anions normally found in blood. Preliminary studies demonstrate the practical application of this system for the determination of salicylate in serum.

As described in the preceding paper [1], initial efforts to utilize membrane dialyzers with potentiometric polymer-membrane electrodes in flow arrangements have focused on designing highly selective detection systems for gases (e.g., $\mathrm{NO}_{x}[1], \mathrm{NH}_{3}$ [2], and $\mathrm{CO}_{2}$ [3]). In these cases, the gas-permeable membrane served to screen out ionic species within the samples which would otherwise interfere with the working electrode. This paper describes how this approach can be used for the detection of neutral organic molecules. The development of an electrode-based flow-injection system suitable for the direct determination of salicylic acid in complex samples is reported.

The determination of salicylate in blood is an important diagnostic test for the detection and prevention of aspirin abuse among patients with chronic

${ }^{a}$ On leave from Sichuan College of Chemical Engineering, Zigong, Sichuan, China. 
pain conditions or in cases of accidental aspirin overdose involving children. Indeed, while therapeutic blood-salicylate concentrations rarely rise above $1.5 \mathrm{mM}$, levels above $2.2 \mathrm{mM}$ are considered toxic [4]. The classical spectrophotometric method for detecting salicylate in blood involves the formation of a complex between salicylate and excess of iron(III) ions [5]. Although relatively simple and fast, this method is subject to many interferences from substances which also form colored complexes with iron(III) [5]. Anionselective liquid-membrane electrodes based on lipophilic ion-exchangers (e.g., Aliquat-336) recently have been proposed for the assay of aspirin in tablets after hydrolysis of the acetylsalicylic acid to salicylate [6]. Unfortunately, these sensors generally suffer significant interference from a wide range of other anionic species (Hofmeister pattern [7]) and thus cannot be utilized for direct measurements in blood where chloride levels are quite high (98$109 \mathrm{mM}[8]$ ).

In earlier work [9], it was observed that commercial carbon dioxide sensors equipped with silicone-rubber gas-permeable membranes displayed rather large responses toward salicylate and other organic anions in acidic solutions. This interference results from the ability of salicylic acid to permeate the silicone-rubber membrane and alter the $\mathrm{pH}$ of the internal electrolyte layer in contact with a glass $\mathrm{pH}$ electrode. Such interference was not observed when microporous teflon membranes were used in place of silicone rubber. These observations suggested that under certain conditions, potentiometric gas-sensing configurations could be useful for detecting relatively large neutral molecules as well as gases. However, because the membrane diffusion rates of the larger organic compounds are considerably slower than those of gases, long response times and even more sluggish recovery times (from high concentration to low) preclude the use of conventional static gas-sensor designs for such purposes.

In the present work, the concept of using a membrane dialyzer as a sample loop in an electrode-based flow-injection arrangement solves this problem by separating the selective molecular dialysis from the down-stream potentiometric sensor. Further improvements in selectivity for one acid over another can be achieved by using a more discriminating indicator electrode as the detector. A schematic diagram of the system designed for salicylate is shown in Fig. 1. A fresh plug of internal recipient buffer is used for each measurement. As illustrated, the upper channel of the dialyzer serves as the injection loop of a conventional flow-injection set-up. Unlike the preceding $\mathrm{NO}_{x}$ system [1], the bottom channel of the dialyzer serves as a second loop into which the test sample is loaded manually as a static plug. Figure 2 shows an expanded view of the dialysis chamber as well as the chemical and physical processes involved. After a fixed trapping time, the solution in the upper channel is flushed to the electrode and the salicylate levels are detected potentiometrically. It will be shown that the use of this membrane-dialyzer/ flow-injection arrangement substantially improves the effective selectivity of the polymer membrane electrode such that salicylate can be determined directly in serum. 


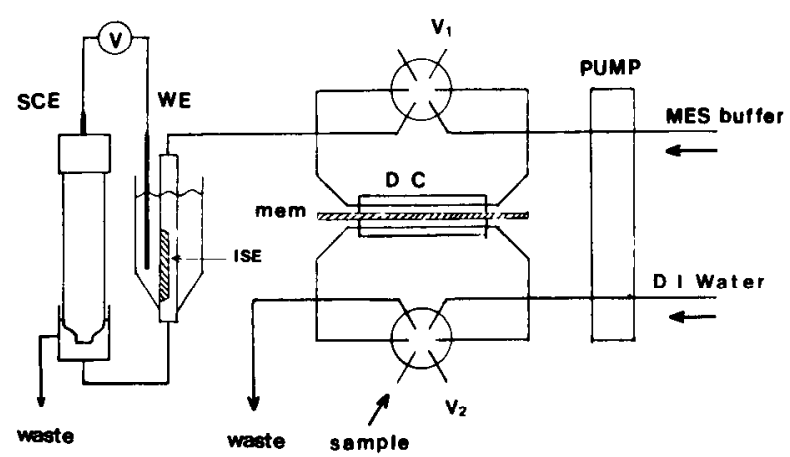

Fig. 1. Schematic diagram of the dialyzer/flow injection set-up for determining salicylic acid; (ISE) tubular PVC ion-selective membrane; (WE) working electrode unit for potentiometric measurements; ( $\mathrm{SCE}$ ) saturated calomel reference electrode; ( $\mathrm{V}$ ) $\mathrm{pH} / \mathrm{mV}$ meter; (DC) dialysis chamber; (mem) silicone rubber membrane; $\left(V_{1}, V_{2}\right)$ flow-injection valves.
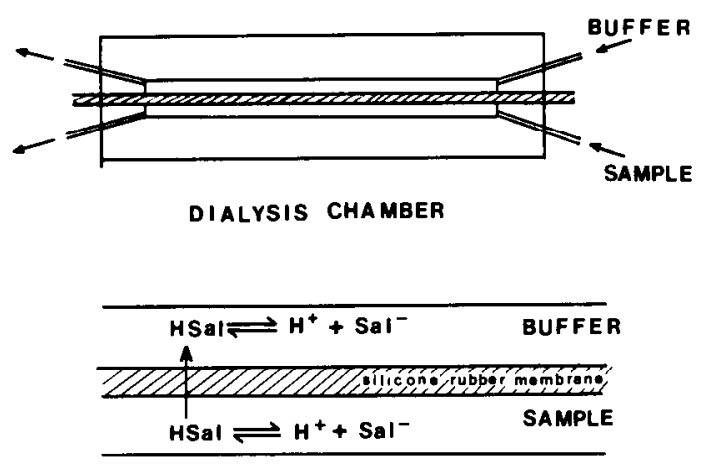

DI ALYSIS

Fig. 2. Expanded view of the membrane-dialyzer unit and outline of the processes which take place therein.

\section{EXPERIMENTAL}

\section{Apparatus}

A schematic diagram of the final flow-injection system is shown in Fig. 1. An AutoAnalyzer proportioning pump, model-1 (Technicon) was used to deliver the carrier solution $\left(3.2 \mathrm{ml} \mathrm{min}^{-1}\right)$ and a water wash $\left(3.2 \mathrm{ml} \mathrm{min} \mathrm{min}^{-1}\right)$ through the membrane dialyzer. The dialysis unit was a special wide-channel type identical to that used in the preceding report [1]. The unit was fitted with a 0.1-mm thick vinyl-methyl silicone rubber membrane (SciMed Life System, Minneapolis, MN). For all the data reported here, the dialysis chamber was submerged in a water bath maintained at $25^{\circ} \mathrm{C}$. Two Rheodyne (model 5020) 4-way rotary injection valves $\left(V_{1}\right.$ and $\left.V_{2}\right)$ were used to introduce the sample and control the dialysis times. All potentiometric measurements were made with a Beckman model 4500 Digital Altex $\mathrm{pH} / \mathrm{mV}$ meter and recorded on a Heath-Schlumberger strip-chart recorder. 


\section{Reagents}

All chemicals were reagent grade. Standard and buffer solutions were prepared with distilled-deionized water.

4-Morpholine-ethanesulfonic acid (MES) was obtained from Aldrich. The materials used in electrode preparation were Aliquat-336 (trioctylmethylammonium chloride; Aldrich), dibutyl sebacate and dioctyl phthalate (Eastman-Kodak) and poly(vinyl chloride) powder (PVC; Scientific Polymer Products, Ontario, NY). The manganese(III) tetraphenylporphyrin chloride (MnTPPCl) used to prepare the porphyrin-based salicylate electrode was a gift from Professor John Groves, Princeton University.

For the porphyrin-based electrode system, $0.05 \mathrm{M}$ MES/sodium hydroxide buffer, $\mathrm{pH}$ 6.5, was used as both the carrier and recipient solution in preliminary characterization studies. For serum measurements, a 0.1 M MES/ sodium hydroxide buffer, $\mathrm{pH} 6.5$, was used. In studies with the Aliquat-based salicylate electrode, a $0.05 \mathrm{M}$ Tris(hydroxymethyl)aminomethane/phosphoric acid buffer, $\mathrm{pH} 8.0$, served as the carrier and recipient solution.

\section{Preparation and characterization of tubular flow-through salicylate electrodes}

Two types of salicylate-sensitive electrodes were investigated. Both were fabricated with $0.89 \mathrm{~mm}$ i.d. PVC tubing in a manner similar to that described for preparation of potassium electrodes [10]. One simplification in the design involved threading the tubular electrode through the small open end of a plastic Finnpipette tip to form a compartment in which the internal reference solution and $\mathrm{Ag} / \mathrm{AgCl}$ electrode can be placed. The tight fit of the electrode tubing within the small opening of the pipette tip formed a natural seal. Once filled with internal reference solution, a piece of Parafilm was used to seal the top of the assembly to prevent slow evaporation of the internal solution.

The Aliquat 336-based electrodes were prepared by using the casting solution described by Choi and Fung [6] for fabrication of their salicylateresponsive electrode. A $0.1 \mathrm{M}$ sodium chloride solution containing $0.01 \mathrm{M}$ sodium salicylate and saturated with silver chloride was used as the internal reference fill. The MnTPPCl-based salicylate electrode was prepared with the following casting solution: $1.2 \mathrm{mg}$ of $\mathrm{MnTPPCl}, 30 \mathrm{mg}$ of PVC powder, and $60 \mu \mathrm{l}$ of dibutyl sebacate, all dissolved in $1 \mathrm{ml}$ of freshly distilled tetrahydrofuran. A $0.05 \mathrm{M}$ MES/sodium hydroxide buffer, $\mathrm{pH} 6.5$, containing $0.01 \mathrm{M}$ sodium salicylate and $0.1 \mathrm{M}$ sodium chloride was used as the internal reference solution.

Evaluation of polymer-membrane electrodes. Before the final dialyzer/ flow-injection system was used, both of the tubular salicylate electrodes were evaluated for response toward salicylate, as well as other anions. This was accomplished by using the appropriate carrier-stream buffer solution for each of the electrodes and directly loading standard solutions of sodium salicylate or other anion salts (prepared in the working buffer) into the flow- 
injection loop provided by the top channel of the dialyzer. The loop was loaded manually by suction with a syringe. This direct-injection mode enabled the performance of the electrodes to be evaluated without changing the working parameters of the final system (e.g., flow rates, pressures and dispersion). Calibration and selectivity data were obtained by measuring the peak height ( $\Delta E$, from the baseline) of the potentiometric signals and plotting these values vs. the logarithm of the anion concentration.

\section{Evaluation of final membrane-dialyzer/flow-injection arrangement}

In these studies, standard solutions of salicylate or other anions were prepared in $0.05 \mathrm{M}$ sulfuric acid. Before introduction of the sample through the valve $\left(V_{2}\right.$ in Fig. 1$)$, both valves were turned to the load position. Thus, in the $V_{1}$ loop, a fresh segment of recipient buffer solution was stopped within the dialyzer channel. The standard solutions were then loaded by suction through valve $V_{2}$ into the lower channel of the dialyzer. The total volume of the loop $\mathrm{V}_{2}$ was $0.68 \mathrm{ml}$ (including connecting tubing), and use of $2 \mathrm{ml}$ of sample ensured that the loop was completely flushed of the previous solution and that the lower dialyzer channel was completely filled with sample. The sample remained in the chamber for a fixed time $(2 \mathrm{~min}$ in most experiments). At the end of this period, both valves were turned to the "inject" position and the recipient plug passed through the tubular electrode to waste. At the same time, the sample solution was flushed from loop $\mathrm{V}_{2}$. Calibration and selectivity data were obtained by measuring the peak height $(\Delta E)$ for each sample as described above.

\section{Procedure for salicylate in serum}

Some unidentified outdated plasma was obtained from the Department of Public Health, Washtenaw County, Michigan. This frozen plasma was thawed, centrifuged to remove any particulate matter, and then stored at $5^{\circ} \mathrm{C}$ until use. Preliminary measurements indicated that the plasma did not contain any measurable salicylate. Thus, it provided a suitable serum-like matrix in which to prepare standards. These standards were prepared by spiking various aliquots of plasma with known amounts of sodium salicylate. Calibration curves were obtained by injecting 1:5 dilutions ( 1 part sample and 4 parts acid) of these standards (in $0.05 \mathrm{M}$ sulfuric acid) into the dialyzer/flow-injection system.

Recoveries were studied on a serum sample drawn from one of the authors who did not previously ingest aspirin. Again, this serum sample initially had an insignificant concentration of salicylate as measured with the electrodebased instrument. Aliquots of this serum sample were spiked with different levels of salicylate. The spiked samples were then diluted 1:5 with acid and the resulting peak potentials were recorded and compared to a two-point working curve prepared with the plasma-based standards. 


\section{RESULTS AND DISCUSSION}

Responses of the electrodes based on MnTPPCl and Aliquat-336

In preliminary studies with the membrane dialyzer system, Aliquat-336 was used as the active membrane component in the salicylate-sensitive polymer electrode described by Choi and Fung [6]. However, results from these feasibility experiments indicated that in order to obtain adequate detection limits for serum, impractically long trapping times would be required. This was due to the relatively poor response of the Aliquat membrane to salicylate in the flow system (see Fig. 3) in combination with a $3-4 \%$ salicylate transfer efficiency within the dialyzer (based on acceptable trapping times, e.g., $2 \mathrm{~min}$ ). Consequently, the possibility of using other ion-exchangers or carriers was investigated to obtain a polymeric salicylate electrode with better detection capabilities.

Separate studies in this laboratory with conventional polymer-electrode designs had indicated that membranes based on MnTPPCl responded to salicylate at very low concentrations when MES buffer, $\mathrm{pH}$ 6.5, was used as the background electrolyte. The response of a tubular flow-through design in which the polymer membrane was prepared with MnTPPCl was then directly compared with the response to salicylate of the Aliquat system. From Fig. 3, it is evident that the porphyrin-based membrane offers a significant improvement in detection limits while maintaining a nearly Nernstian slope (typically 60-64 mV/decade) over a wide concentration range $\left(2 \times 10^{-6}-10^{-2} \mathrm{M}\right)$. Fresh MnTPPCl-based electrodes often had even higher slopes (e.g., 70$80 \mathrm{mV}$ ) but this super-Nernstian behavior usually disappeared after the first day of use. It should be noted that the data shown in Fig. 3 were obtained with the same flow-injection set-up, but with different background electrolyte buffers (carrier solutions). Separate experiments indicated that these background electrolyte buffers yielded the optimum response to salicylate
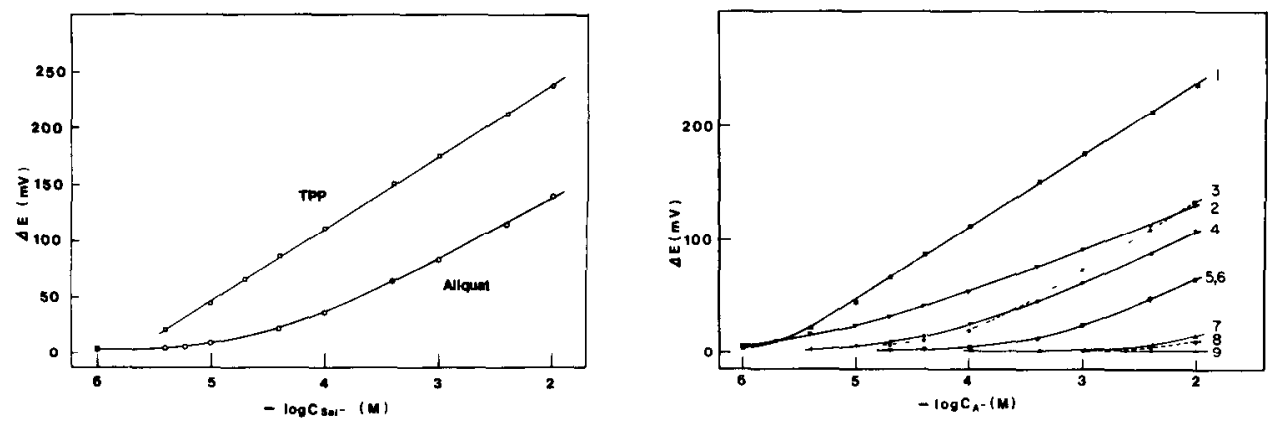

Fig. 3. Responses of the electrodes based on MnTPPCl and Aliquat-336 to salicylate in appropriate buffers in the f.i.a. arrangement.

Fig. 4. Response of the MnTPPCl membrane to various anionic species in MES/NaOH buffer, pH 6.5 : (1) salicylate; (2) $\mathrm{ClO}_{4}^{-}$; (3) acetylsalicylate; (4) benzoate; (5) $\mathrm{Cl}^{-} ;(6) \mathrm{Br}^{-}$; (7) lactate; (8) $\mathrm{HCO}_{3}^{-} ;(9)$ oxalate. 
for the respective electrode systems (i.e., the Aliquat-based electrode had much worse detection limits when evaluated in the $0.05 \mathrm{M} \mathrm{MES/sodium}$ hydroxide, $\mathrm{pH} 6.5$, than in the Tris buffer). Based on these experiments, the porphyrin electrode was used in all subsequent studies.

Aside from improved detection capabilities, the MnTPPCl-based membrane also exhibited an interesting selectivity pattem toward other anionic species. Figure 4 shows the potentiometric response of this membrane to salicylate as well as other inorganic and organic ions in the flow system. It was found that most of the response to acetylsalicylic acid was due to the presence of salicylate in the standards as a result of hydrolysis of the test compound in the $\mathrm{pH}$ 6.5 MES/sodium hydroxide buffer. Overall, the response pattern closely resembles, but is not identical to, the Hofmeister sequence most often observed for anion-responsive liquid-membrane electrodes based on ionexchangers [7]. For example, response to bromide and chloride is essentially the same, yet the Hofmeister sequence predicts greater response to bromide. In addition, response slopes for certain anions (e.g., perchlorate) are considerably sub-Nernstian over a rather wide concentration range. Further, in separate experiments (not shown), it was found that the MnTPPCl membrane has a rather large response to hydroxide even at hydroxide levels approaching $10^{-9} \mathrm{M}$. This mandates the use of a relatively strong buffer when this electrode is applied. While the exact mechanism of ion extraction and transport by the MnTPPCl within the membrane is not clear, it is believed that the porphyrin behaves as an ion-exchanger or neutral carrier (depending on whether the fifth axial ligand is neutral or anionic) to which salicylate can coordinate as a sixth axial ligand with the manganese center. Recent studies by Schulthess et al. [11] on a new membrane electrode for nitrite based on the use of a Vitamin $B_{12}$ derivative support this hypothesis.

While the MnTPPCl electrode displayed desirable detection limits and an unusual selectivity pattern, its useful lifetime was much less than that of the Aliquat-based electrodes. Indeed, with constant use over a 1-week period, the baseline potential continuously became less positive, and detection limits for salicylate gradually became poorer. Therefore, the MnTPPCl membrane was typically replaced every $7-10$ days.

Response characteristics and selectivity of the dialyzer/flow-injection system for salicylic acid

When acidified salicylate samples are loaded into the lower channel of the dialyzer, the salicylic acid molecule partitions into the silicone rubber (Fig. 2) and diffuses through the membrane. This process is aided by the buffer trap (MES/sodium hydroxide, $\mathrm{pH} \mathrm{6.5)} \mathrm{provided} \mathrm{by} \mathrm{the} \mathrm{fresh} \mathrm{recipient} \mathrm{solution} \mathrm{in}$ the upper channel. This solution traps the salicylic acid as salicylate, thereby maintaining a large chemical potential gradient of salicylic acid across the membrane. Figure 5 shows a typical strip-chart recording obtained with this system for aqueous salicylic acid standards and a 2-min trapping time. It can be seen that, despite the manual operation of the valves, reproducibility of 

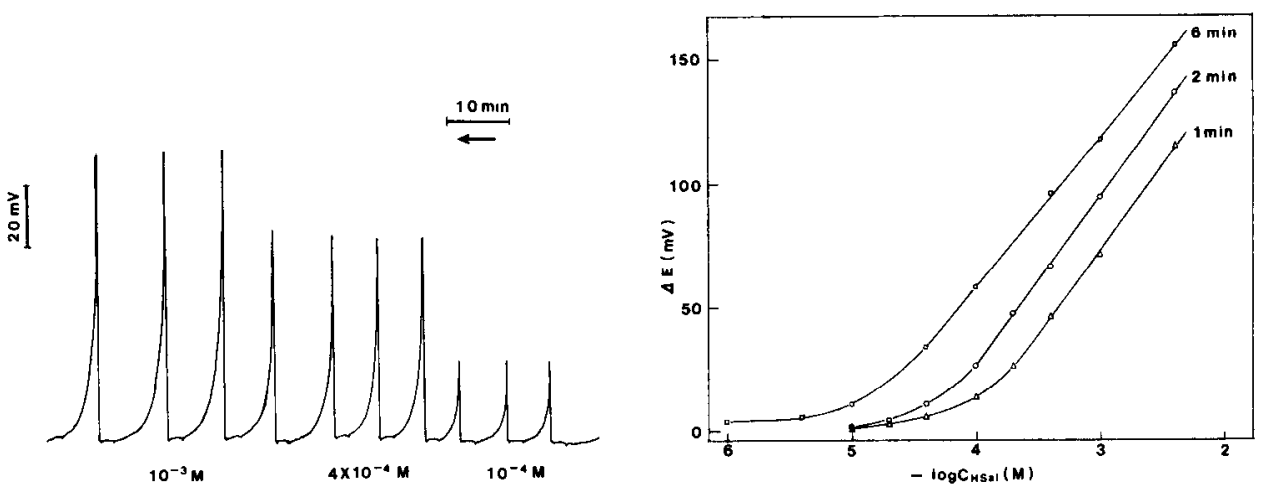

Fig. 5. Typical strip-chart recording obtained from the dialyzer/flow-injection arrangement for replicate injections of aqueous salicylic acid standards using 2-min trapping times.

Fig. 6. Effect of trapping time $(1-6 \mathrm{~min})$ on the response of the dialyzer/flow-injection arrangement to salicylic acid.

the peak heights is good (standard deviations for triplicate injections of the same standards are typically about $1.4 \mathrm{mV}$ ). However, it can also be seen that the baseline recovery time after each sample injection is rather long. These tailing peaks are caused by the reservoir of salicylic acid left behind in the silicone membrane (which continues to leach out even after injection) as well as the inherently slow recovery times of the porphyrin-based membrane. The latter was observed in the direct-injection experiments undertaken to characterize the tubular membrane electrode and can be attributed to a high association constant between the salicylate anions and the porphyrin within the membrane.

Naturally, for given salicylic acid concentrations, the magnitude of the potentiometric response was governed by the trapping time. Figure 6 shows how the detection limits of the system can be improved by using a longer trapping period. For the 2-min trapping time used in much of this work, the efficiency of salicylic acid transfer from the sample to the recipient solution was calculated to be typically $4-7 \%$, based on prior direct-injection calibration of the salicylate electrode. This trapping efficiency increased when the dialyzer was maintained at higher temperatures; however, elevated temperatures tended to cause outgassing of the solutions and bubble problems. Thus, all experiments were done with the dialyzer thermostatted at $25^{\circ} \mathrm{C}$. At this temperature, there usually is a slight increase in dialysis efficiency with increasing concentration of salicylic acid and this effect causes somewhat super-Nernstian behavior in the final sensing arrangement (e.g., slopes are generally $64-70 \mathrm{mV} /$ decade) even when the tubular $\mathrm{MnTPPCl}$ electrode itself has Nernstian response properties. This type of behavior has been observed previously with gas sensing dialyzer systems $[2,12]$.

The selectivity of the final membrane-dialyzer/flow-injection arrangement 

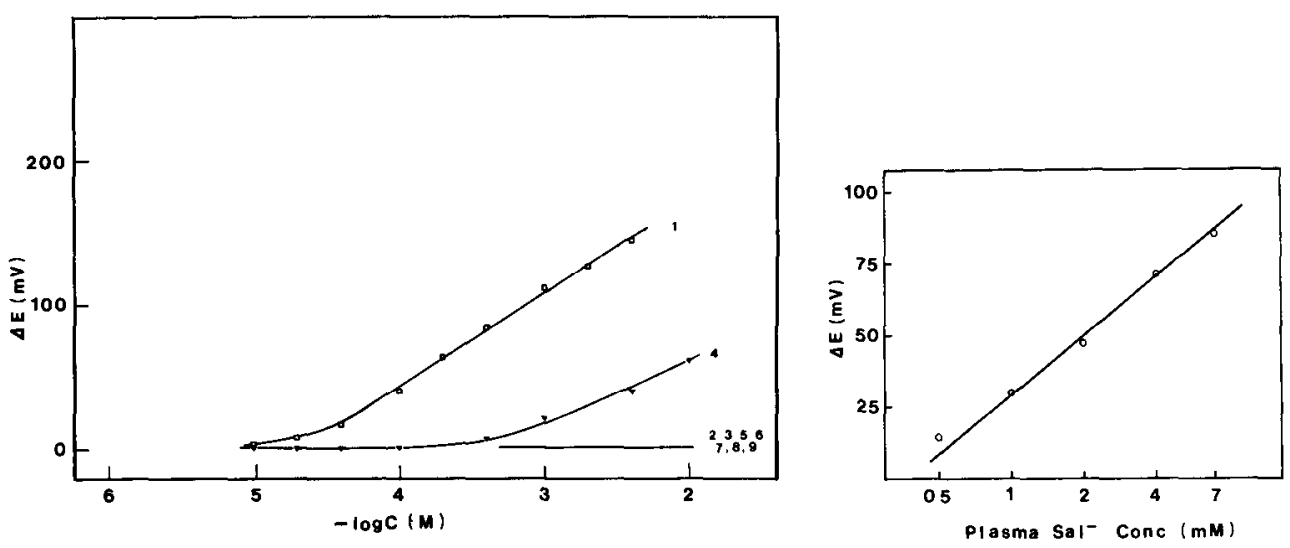

Fig. 7. Potentiometric response of the dialyzer/flow-injection arrangement to various species in acid using a 2-min trapping time. Numbers refer to the anions in Fig. 4.

Fig. 8. Calibration curve obtained for plasma-based salicylate standards. (Plotted as concentration of salicylate in plasma prior to dilution with acid.)

is excellent. As shown in Fig. 7, most of the inorganic and organic ions tested yielded no response at $10^{-2} \mathrm{M}$ concentrations. Even higher levels of chloride $\left(10^{-1} \mathrm{M}\right)$ also resulted in negligible potentiometric response. Comparison of these results with those in Fig. 4 clearly illustrates how the silicone-rubber membrane effectively eliminates anionic interferences for those species which cannot permeate this membrane. For example, perchlorate, which is a major interferent with the porphyrin-membrane electrode alone (see Fig. 4) apparently does not get through the silicone-rubber membrane, at least not during the 2-min trapping period. Among the organic acids tested, only benzoic acid represents a significant interference, although the system is more responsive to salicylic acid by a factor of nearly 60 . In this case, the selectivity pattern closely parallels that obtained by direct injection through the membrane electrode because both species probably diffuse through the silicone rubber membrane at nearly the same rate. Thus, in practice, ultimate selectivity is dictated by the kinetics of transfer through the silicone membrane and the intrinsic response of the detecting electrode toward the anionic form of the species.

\section{Salicylate in serum}

Preliminary efforts to determine the salicylate content of serum samples with aqueous salicylate standards proved difficult. Recovery studies with salicylate-spiked serum consistently yielded recoveries between 60 and $70 \%$ despite the fact that the samples were first diluted 1:5 with acid prior to being loaded into the membrane dialyzer. Clearly, the protein content of the serum was affecting the efficiency of salicylic acid transfer across the siliconerubber membrane. To overcome this matrix effect, salicylate standards were 
TABLE 1

Summary of salicylate results for serum

Salicylate conc. (mM)

\begin{tabular}{lllllr}
\hline Added & Found & & & \multicolumn{2}{c}{ Recovery (\%) } \\
\cline { 2 - 3 } & Day 1 & Day 2 & & Day 1 & Day 2 \\
\hline 1.00 & 0.96 & 1.01 & & 96 & 101 \\
2.00 & 1.72 & 1.96 & 86 & 98 \\
4.00 & 4.22 & 3.59 & 105 & 90 \\
\hline
\end{tabular}

a From a 2-point calibration made before test. ${ }^{b}$ Values are the average of two determinations.

prepared in a plasma matrix. Figure 8 shows a typical working curve obtained with these standards. It can be seen that the plot is linear over the range of salicylate concentrations required for blood measurements [4]. A two-point calibration in this range was used in a further recovery study of spiked serum samples. Table 1 shows that recoveries ranged from 86 to $105 \%$ for two separate experiments. In view of the uncertainties associated with manual control of the trapping time as well as the time needed for manual loading of the sample, these results are considered satisfactory so that the proposed method can be used to estimate salicylate concentrations in serum. Fully automated arrangements would obviously provide better precision.

The authors gratefully acknowledge the National Institutes of Health for supporting this work (Grant GM-28882-05).

\section{REFERENCES}

1 G. B. Martin and M. E. Meyerhoff, Anal. Chim. Acta, 186 (1986) 71.

2 Y. M. Fraticelli and M. E. Meyerhoff, Anal. Chem., 53 (1981) 992.

3 W. N. Opdycke, S. J. Parks and M. E. Meyerhoff, Anal. Chim. Acta, 155 (1983) 11.

4 R. V. Blanke, in N. W. Teitz (Ed.), Fundamentals of Clinical Chemistry, Saunders, Philadelphia, 1976, Chap. 21.

5 P. Trinder, Biochem. J., 57 (1954) 301.

6 K. K. Choi and K. W. Fung, Anal. Chim. Acta, 138 (1982) 385.

7 W. E. Morf, The Principles of Ion-Selective Electrodes and of Membrane Transport, Elsevier, Amsterdam, 1981, Chap. 11.

$8 \mathrm{~N}$. Weissman and V. J. Pileggi, in R. J. Henry, D. C. Cannon and J. W. Winkelman (Eds.), Clinical Chemistry, Principles and Technics, Harper and Row, Hagerstown, MD, 1974, Chap. 19.

9 R. K. Kobos, S. J. Parks and M. E. Meyerhoff, Anal, Chem., 54 (1982) 1976.

10 P. M. Kovach and M. E. Meyerhoff, J. Chem. Educ., 60 (1983) 766.

11 P. Schulthess, D. Ammann, B. Krautler, C. Caderas, R. Stepánek and W. Simon, Anal. Chem., 57 (1985) 1397.

12 H. L. Lee and M. E. Meyerhoff, Analyst, 110 (1985) 371. 